\title{
Comparison of iPTH values in serum and plasma samples depending on the time and temperature in patients with chronic kidney disease
}

\author{
Bencova $\mathrm{V}^{1}$, Maris $\mathrm{K}^{1}$, Spustova $\mathrm{V}^{2}$, Gazovic $\mathrm{V}^{3}$, Straussova $\mathrm{Z}^{4}$, Cernohorska $\mathrm{B}^{5}$, Nemethova $\mathrm{D}^{6}$
}

DIA-NE, s.r.o., Nitra. vbencova@gmail.com

\begin{abstract}
Renal osteodystrophy is a systemic disorder associated with chronic kidney disease (CKD) with abnormal values of biochemical parameters related to bone and mineral metabolism. Assessing renal osteodystrophy subtypes is especially important for diagnostic and therapeutic decision. Management of these disorders includes monitoring of homeostasis of calcium, phosphorus and parathormone (PTH). PTH is a significant regulator of mineral balance and it's level is used as a surrogate biomarker for the type of underlying renal osteodystrophy. Worldwide, nephrologists rely on KDIGO - Clinical Practice Guideline for the Diagnosis, Evaluation, Prevention, and Treatment of Chronic Kidney Disease - Mineral and Bone Disorder (CKD-MBD) to maintain PTH levels within defined narrow range of optimal values for each stage of CKD and adjust such PTH - lowering treatments as active vitamin D sterols or calcimimetics accordingly. PTH is rapidly degraded in vivo, with half life of 5 minutes and it is also unstable in blood samples. Values can differ significantly when samples are not collected in a standard way and when recommended conditions for transport and sample processing are not followed. It is also important to standardize pre-analytic conditions that may influence the variability in PTH results. The goal of the present study was to compare iPTH stability in serum and plasma samples and evaluate possible pre-analytic errors in sample collection, effect of temperature during transportat and storing prior to analysis (Tab. 1, Fig. 1, Ref. 5). Text in PDF www.elis.sk. Key words: parathormone, chronic kidney disease, pre-analytic errors.
\end{abstract}

Circulating parathyroid hormone (PTH) should be measured regularly in patients with chronic kidney disease (CKD) to diagnose and evaluate secondary hyperparathyroidism and CKD related mineral and bone disorder. PTH level is often used as a surrogate biomarker for the type of underlying osteodystrophy and for adjustment of PTH-lowering treatments.

Parathyroid hormone (PTH) plays a key role as a systemic regulator of calcium, phosphate and vitamin D metabolites in blood. It is also a major regulator of cellular activity in bone. Classical biological activities of PTH are mediated by PTH1R receptors, which are present in some tissues, while the amino- terminal region of the molecule contains the sequence necessary to active PTH1R. PTH present in circulation is very heterogeneous, and this is a consequence of a complex metabolism (1). PTH is a linear peptide of 84 amino acids, produced in the parathyroid glands, encoded in humans by a gene located on the short arm of chromosome 11 . The transcription product not only encodes the 84 amino acids of the final peptide, but also encodes "pre" signal sequence

${ }^{1}$ DIA-NE, s.r.o., Nitra, ${ }^{2}$ Slovak Medical University, Bratislava, ${ }^{3} \mathrm{G}-\mathrm{LAB}$, s.r.o., Nitra, ${ }^{4} \mathrm{FMC}$ - dialyzačné služby, s.r.o., Nitra, ${ }^{5} \mathrm{FMC}$ - dialyzačné služby, s.r.o., Piešt’any, Slovakia, ${ }^{6}$ Institute of Biostatistics and Analyses Faculty of Science and Faculty of Medicine Masaryk University, Brno, Czech Republic

Address for correspondence: V. Bencova, MD, Panska dolina 41, SK94901 Nitra, Slovakia. of 25 amino acids and basic "pro" hexapeptide. The "pre" signal sequence plays a role in transport to endoplasmic reticulum and "pro" hexapeptide sequences hydrolyzed by endopeptidases in the Golgi apparatus. The resulting final 84 -amino acid hormone is subsequently packed into secretory granules and transported to cell membrane. Metabolism of PTH is regulated by ionized calcium concentration in blood. PTH secretion is controlled by the extracellular calcium-sensing receptor (CaSR) and $\mathrm{G}$ protein-coupled receptor on the surface of parathyroid glands. The inhibition of PTH (1-84) secretion is accompanied by an enhanced proteolysis with secretion of other peptide fragments, PTH (7-84) (2). PTH (1-84) and variety of derived peptide fragments are present simultaneously in peripheral blood. Indeed, the concentration of peptide fragments is several-fold greater than the concentration of intact hormone.

PTH is rapidly degraded in vivo, with the half life of 5 minute and it is also unstable in blood samples (3). For the correct measurement of intact PTH, the correct handling of the blood specimens is of crucial importance.

\section{The aim of the study}

To compare iPTH stability in serum and plasma samples. To evaluate possible pre-analytic errors, such as collection of samples and the effect of temperature during transportation and storing prior to analysis. 


\section{Methods}

Serum and plasma samples from 100 dialyzed patients were studied. Four serum samples and 4 plasma samples from each patient were analyzed. Control serum and control plasma samples from individual patients were collected, stored, transported at the temperature of $4{ }^{\circ} \mathrm{C}$ and analyzed within 2 hours since the collection according to the standard recommendation. Other 3 serum samples and 3 plasma samples were collected and transported to the laboratory at the room temperature and analyzed after 2 hours, 6 hours and 24 hours since collection. The concentration of iPTH was determined by Elecsys 2010 auto-analyser (Roche Diagnostics, Germany).

\section{Statistical analysis}

The analysed data set contained data measured in 100 patients. For each patient 8 values of PTH were recorded. The values of PTH were identified as log-normally distributed and hence (for better statistical characteristics) transformed using the logarithmic transform: $x^{\prime}=\log 10(\mathrm{x})$. Normality of the transformed values was tested and confirmed by the Kolmogorov-Smirnov test. Such data transformation and the consequent Kolmogorov-Smirnov test enabled us to analyse the data using parametric statistical tests. For the comparison of the PTH values according to the reference samples (kept on ice) from serum and plasma, the paired t-test was used. For the evaluation of difference in the values of PTH level among the four blood serum samples (one reference sample kept on ice and three samples kept at the room temperature), the repeated measures ANOVA was used. Moreover, we used also Dunnett's test for the assessment of difference between the room temperature samples and reference sample. The same methods were also applied for the evaluation of differences among the recorded values of PTH level extracted from plasma.

For all statistical considerations, the significance level $\alpha=$ 0.05 was used.

\section{Results}

iPTH values in control serum and control plasma did not differ significantly when collected in a standard way and when recommended conditions for transportation and samples processing were followed. A significant difference in iPTH values was found in serum samples, which were not stored according to standards. The results are described in the Table 1 and Figure 1.

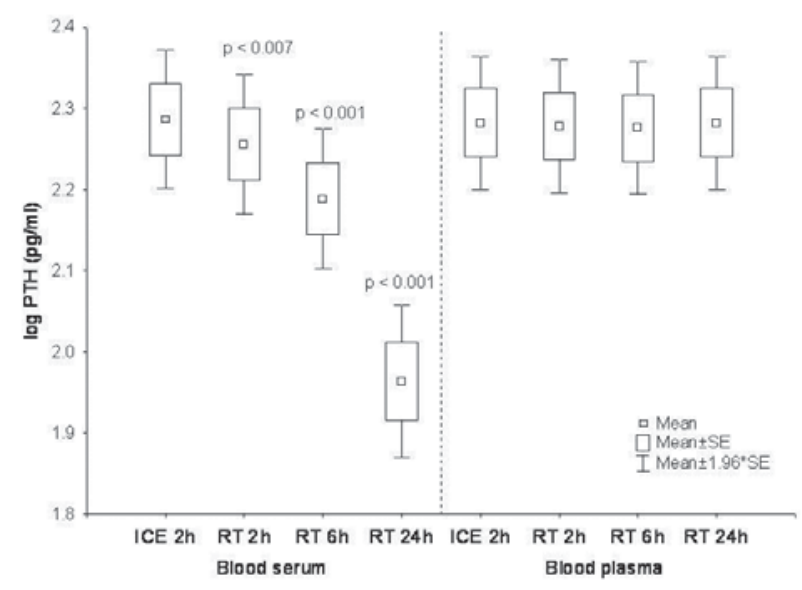

Fig. 1. Logarithmically transformed values of parathormone level (PTH) according to the origin of sample (serum, plasma). PTH: parathormone, S - Ice: reference sample from serum kept on ice, S - RT: samples from serum kept at the room temperature, $P$ - Ice: reference sample from plasma kept on ice, $\mathrm{P}$ - RT: samples from plasma kept at room temperature, Abbreviations $2 \mathrm{~h}, 6 \mathrm{~h}, 24 \mathrm{~h}$ refer to time from the moment of blood collection, when the PTH level was measured.

\section{The comparison of the reference samples}

The difference between PTH level recorded in the reference serum and plasma samples could not be proven as statistically significant (paired t-test: $\mathrm{t}=0.970, \mathrm{df}=99, \mathrm{p}<0.334$ ). Thus, the starting PTH level was observed to be the same for both types of blood samples.

\section{The serum}

It was confirmed that the difference among values of PTH level measured in four serum samples at different room temperatures was statistically significant (Repeated measures ANOVA: F $=422.062, \mathrm{df} 1=3, \mathrm{df} 2=297, \mathrm{p}<0.001)$. For the assessment of difference in the PTH level between the serum reference sample and three samples taken at the room temperature, the Dunnett's post-hoc test was used. All three samples taken at room temperature have shown a lower PTH level than the reference sample (Tab. 1 and Fig. 1).

The sample kept at room temperature and evaluated after 2 hours from the blood collection had shown a $6.5 \%$ decrease (in the mean) of the PTH level with respect to the reference sample kept on ice. The other sample kept at room temperature and evaluated

Tab. 1. Parathormone level (pg/ml) evaluated in serum and plasma samples after 2, 6 and 24 hours from the time of blood collection.

\begin{tabular}{|c|c|c|c|c|c|c|c|c|}
\hline & $\mathrm{n}$ & Mean & Median & Minimum & Maximum & $10 \%$ quantile & $90 \%$ quantile & Standard deviation \\
\hline Serum - Ice $2 \mathrm{~h}$ & 100 & 314.8 & 189.8 & 19.0 & 2575.0 & 57.2 & 655.4 & 382.2 \\
\hline Serum - RT 2h & 100 & 297.4 & 174.8 & 19.8 & 2515.0 & 57.1 & 621.2 & 367.3 \\
\hline Serum - RT 6h & 100 & 256.7 & 148.5 & 15.6 & 2220.0 & 49.3 & 511.9 & 323.6 \\
\hline Serum - RT 24h & 100 & 167.3 & 92.4 & 5.6 & 1537.0 & 21.4 & 343.5 & 231.0 \\
\hline Plasma - Ice $2 \mathrm{~h}$ & 100 & 302.4 & 180.8 & 22.4 & 2287.0 & 62.2 & 603.6 & 350.8 \\
\hline Plasma - RT 2h & 100 & 300.2 & 175.8 & 23.9 & 2122.0 & 62.5 & 607.8 & 344.5 \\
\hline Plasma - RT 6h & 100 & 297.7 & 176.1 & 23.1 & 2196.0 & 56.6 & 584.7 & 341.2 \\
\hline
\end{tabular}


after 6 hours from the blood collection has shown a $19.4 \%$ decrease of the PTH level with respect to the reference sample kept on ice and a $13.9 \%$ decrease of the PTH level with respect to the sample kept at the room temperature evaluated after 2 hours from the blood taking. When considering the room temperature sample evaluated after 24 hours from the blood collection, the values of PTH level had been $49.8 \%$ lower (in the mean) according to the reference sample, $46.7 \%$ lower according to the sample evaluated after 2 hours from the blood collection and $38.6 \%$ lower according to the sample evaluated after 6 hours from the blood collection.

\section{The plasma}

It was not statistically proven that the difference in the PTH level between the four considered plasma samples was statistically significant (Repeated measures ANOVA: $\mathrm{F}=2.468$, df1 $=$ 3 , df2 $=297, \mathrm{p}<0.062$ ).

\section{Conclusion}

There was no statistically significant difference in the PTH level between the serum and plasma reference samples.

Parathormone levels were significantly lower in the serum samples kept at the room temperature after blood collection than in the serum reference sample. This implies that the PTH molecules present in the serum sample were not stable in time when not kept at standard conditions (on ice).

On the other hand, no significant difference in the PTH level was observed for plasma samples, which implies that the plasma samples are stable in time even when kept at the room temperature.

It can be concluded that determination of $\mathrm{PTH}$ in plasma is not jeopardized by possible error in pre-analytic phase of examination, which can have significant consequences for the diagnosis and treatment of bone disease in patients with chronic kidney failure.

\section{Discussion}

In the present study, we measured circulating PTH concentration in serum and plasma in patients with CKD stage 5 . We found that PTH concentration in serum and EDTA plasma differed significantly regardless the time at the room temperature. The PTH degradation was significant in serum samples, concentration of PTH felt nearly to half after 24 hours at the room temperature. This pre-analytic phase of examination is very important for the final measurement. The investigations showed that EDTA plasma is a good choice based on an excellent PTH stability (4). Our study has some limitations. We didn't compare other PTH assays, used only Roche Elecsys for evaluation of samples. Joly et a. showed that PTH concentration may vary widely depending on the assay used to assess PTH (5).

Regardless of the daily practice in PTH measurement and blood sample collection and handling in dialysis centres, we recommend the use of EDTA plasma for the evaluation.

\section{References}

1. Vieira JGH, Kunii I., Nishida S. Evolution of PTH assays. Arg Bras Endocrinol Metab 2006; 50: 621-627.

2. Friedman PA, Goodman WG. PTH(1-84)/PTH(7-84): a balance of power. Am J Physiol Renal Physiol 2006; 290: F975-F984.

3. Cantor T. The importance of correct specimen preparation and transport in the accurate measurement of PTH. Dial Transplant 2003; 32 (11): $711-718$.

4. Joly D, Drueke TB, Alberti C, Houllier P, Lawson-Body E, Martin KJ, Massart C, Moe SM, Monge M, Souberbielle JC. Variation in serum and plasma PTH levels in second-generation assays in hemodialysis patients: a cross-sectional study. Am J Kidney Dis 2008; 51: 987-995, doi:10.1053/j.ajkd.01.017.

5. Souberbielle JC, Boutten A, Carlier MC, Chevenne D, Coumaros G, Lawson-Body E, Massart C, Monge M, Myara J, Parent X, Plouvier E, Houillier P. Working group on PTH and vitamin D, Société Francaise de Biologie Clinique (SFBC). Kidney Int 2006; 70: 345-350.

Received September 20, 2012. Accepted March 11, 2013. 